\title{
Towards a one-step method for preparing silica/polymer heterodimers and dimpled polymer particles
}

\author{
Isabelle Chaduc, ${ }^{a}$ Julien Parvole, ${ }^{a}$ Tristan Doussineau, ${ }^{b}$ Rodolphe Antoine, ${ }^{b}$ Anthony Désert, ${ }^{c}$ \\ Pierre-Yves Dugas, ${ }^{a}$ Serge Ravaine, ${ }^{d}$ Etienne Duguet, ${ }^{c}$ Elodie Bourgeat-Lami, ${ }^{a}$ Muriel Lansalot*,a \\ a Université de Lyon, Univ. Lyon 1, CPE Lyon, CNRS UMR 5265, Laboratoire de Chimie Catalyse Polymères \\ et Procédés (C2P2), LCPP group, F-69616 Villeurbanne, France \\ ${ }^{b}$ Université de Lyon, Université Lyon 1, CNRS, UMR 5579 LASIM, F-69616 Villeurbanne, France \\ ${ }^{c}$ CNRS, Univ. Bordeaux, ICMCB, UPR 9048, F-33600 Pessac, France \\ ${ }^{d}$ CNRS, Univ. Bordeaux, CRPP, UPR 8641, F-33600 Pessac, France
}

\begin{abstract}
Silica/PS and silica/PMMA heterodimers were obtained in very good yields using $80 \mathrm{~nm}$ silica particles first functionalized with a polymerizable alkoxysilane and then used as seeds for the emulsion polymerization of either styrene or methyl methacrylate. Aiming at a one-pot and scalable process, a commercial silica sol was used and the grafting reaction of silica with the functional silane was directly performed in the mixture of surfactants subsequently used for the polymerization. The effect of silica content, nature and concentration of the surfactant, of the reactive alkoxysilane or of the monomer, on the formation of the silica/PS heterodimers was investigated. Either dumbbell- or snowman-like dimers were obtained depending on monomer conversion. The typical morphological yield reached $60 \%$ with respect to all morphologies. The synthesis of silica/PMMA dumbbell-like dimers was also successful. In addition to TEM and DLS, the emerging technique of charge detection mass spectrometry (CD-MS) was used to provide the true mass distribution and the sample composition in terms of morphologies. Finally, this system also proved to be efficient for the synthesis of dimpled polymer particles.
\end{abstract}

\section{Introduction}

In recent years many efforts have been devoted to the synthesis of colloidal clusters of spherical particles for both fundamental studies and preparation of new materials. ${ }^{1-4}$ In particular, anisotropic clusters constituted of both organic and inorganic particles have been the topic of many studies and 
different processes both physicochemical and chemical have been developed and a vast range of morphologies obtained. ${ }^{5-19}$ A few years ago we reported a one-pot seeded-growth emulsion polymerization process allowing the nucleation and growth of a controlled number of polystyrene (PS) latex nodules onto the surface of silica seed particles. ${ }^{20}$ To promote the affinity between both phases, the surface of the silica seed was previously treated by an alkoxysilane bearing a polymerizable group. The idea was to promote the capture of the growing chains initiated in water by copolymerizable species located at the surface of the silica seed, and thus favour the nucleation/growth of the polymer nodules onto the seed. According to this strategy various morphologies have been observed such as dumbbell- or snowman-like dimers, ${ }^{21}$ and multipod-like clusters. ${ }^{20,}{ }^{22}$ Various parameters such as the concentration and nature of the compatibilizing agent, the nature and concentration of the surfactant, the size and concentration of the silica seed or the grafting density of the silane molecules proved to strongly impact the final morphology.

To date, the synthesis of heterodimers (either snowman- or dumbbell-like) has been limited to low monomer conversions and the influence of the experimental conditions on the control of the morphology has not been studied in details. Aiming at a scalable and robust process allowing the formation of a large quantity of dissymmetrical particles in one step, the original process ${ }^{21}$ has been modified and improved in many ways. Within this work, a commercial silica sol was used instead of home-made silica seeds. In place of common hydroalcoholic protocols, the grafting reaction was performed directly in water in the presence of the surfactants subsequently used for the emulsion polymerization, providing the opportunity to skip time-consuming steps of separation/redispersion of the silica in water. To improve both the polymerization kinetics and the colloidal features of the polymer nodules (stability and dispersity), an anionic surfactant was added to the non-ionic one used in the initial recipe. Using this new protocol, the effect of various experimental parameters (silica content, nature and concentration of the surfactants, alkoxysilane and monomer) on the formation of silica/PS dimers was investigated. The formation of silica/poly(methyl methacrylate) (PMMA) dimers is also reported for the first time using alkoxysilane-modified seeds. The final silica/polymer dimers were characterized by using conventional transmission electron microscopy (TEM) and dynamic light scattering (DLS) techniques. A very innovative aspect of this work is that the emerging technique of charge detection mass spectrometry $(C D-M S)^{23}$ was used as a complementary tool for the characterization of the silica/polymer dimers ${ }^{24}$ to clear any doubt about the sample composition in terms of cluster morphology. Finally, this system also revealed to be an efficient strategy for the synthesis of dimpled polymer particles. 


\section{Experimental}

Materials. Styrene (Sty, 99\% from Acros) was purified by passing over a column of basic aluminum oxide. Methyl methacrylate (MMA, 99\% from Acros), sodium dodecyl sulfate (SDS, 99\% from Aldrich), Synperonic ${ }^{\circledR}$ NP30 (NP30, from Fluka), sodium persulfate (NaPS, 98\% from Acros), methacryloxy methyl triethoxysilane (MMS) and methacryloxy propyl dimethyl methoxysilane (MPDMS, 92\% from ABCR) were used without further purification. Commercial silica particles (Klebosol 30R50, AZ Electronic Materials, $32 \mathrm{wt} \%)$ were first centrifugated at $15000 \mathrm{rpm}$ for 15 minutes to remove the very small particles (diameter $\leq 50 \mathrm{~nm}$ ) present in the sample. The number- and mass average particle diameter $\left(D_{\mathrm{n}}\right.$ and $D_{\mathrm{w}}$, respectively) as well as the particle-diameter dispersity $\left(D_{\mathrm{w}} / D_{\mathrm{n}}\right)$ of the silica seeds after centrifugation were determined from TEM observations $\left(D_{\mathrm{n}, \text { silica }}=80 \mathrm{~nm}, D_{\mathrm{w}} / D_{\mathrm{n}}=1.02\right)$. Water was deionized before use (Purelab Classic UV, Elga LabWater). All chemical structures are shown in the Supporting Information (Scheme S1).

Surface modification of the silica seed. The surface of the silica seeds was treated by grafting MMS or MPDMS directly in water. The silane (0.1 function $\left.\mathrm{nm}^{-2}\right)$ was added to the silica suspension containing $\mathrm{NP30}$ and/or SDS and the mixture $(\mathrm{pH}=8.5)$ was stirred at room temperature overnight.

Emulsion polymerization. Styrene or MMA was added to the silane-modified silica suspension. The mixture was introduced in a $250 \mathrm{~mL}$ glass thermostated reactor fitted with a condenser and purged with nitrogen under stirring $(275 \mathrm{rpm})$ for $30 \mathrm{~min}$. It was then heated to $70{ }^{\circ} \mathrm{C}$ and NaPS $(0.5 \mathrm{wt} . \%$ relative to the monomers) dissolved in $1 \mathrm{~mL}$ of deionized water was added to start the emulsion polymerization. The regular withdrawal of samples allowed us to follow the monomer conversion with time by gravimetric analysis. The various polymerizations performed for this study are presented in Table 1.

Formation of dimpled PS particles. A polymerization experiment was carried out following a D8-type recipe (run D11, see Table 1), stopped after $35 \mathrm{~min}$ (corresponding to $34 \%$ styrene conversion). The sample was then gently stirred for one week under nitrogen bubbling. 
Table 1: One-pot seeded-growth emulsion polymerization of styrene or MMA using silane-functionalized silica seeds ${ }^{a}$

\begin{tabular}{|c|c|c|c|c|c|c|c|c|c|c|}
\hline Run $^{a}$ & $\begin{array}{c}{[\mathrm{M}]} \\
\mathrm{g} \mathrm{L}^{-1 b}\end{array}$ & $\begin{array}{c}\text { NP30/SDS } \\
(w / w)\end{array}$ & $\begin{array}{l}\text { [Surf] } \\
\mathrm{g} \mathrm{L}^{-1 \mathrm{c}}\end{array}$ & Silane & $\begin{array}{c}\text { [Silica] } \\
\mathbf{g ~ L}^{-1}\end{array}$ & $\begin{array}{c}\text { Time } \\
\text { (h) }\end{array}$ & $\begin{array}{l}\text { Conv } \\
\text { (\%) }\end{array}$ & $\begin{array}{c}D_{n, \text { PS or PMMA }} \\
(\mathrm{nm})^{\mathrm{d}}\end{array}$ & $D_{\mathrm{w}} / D_{\mathrm{n}}$ & $\begin{array}{l}N_{\text {Latex }} / \\
N_{\text {Silica }} \text { e }\end{array}$ \\
\hline \multicolumn{11}{|c|}{ Styrene emulsion polymerization } \\
\hline D1 & 100 & $100 / 0$ & 3 & MMS & 10 & 2 & 18 & 103 & 1.12 & 1.8 \\
\hline D2 & 100 & $0 / 100$ & 2 & MMS & 10 & 3 & 93 & 65 & 1.10 & 3.6 \\
\hline D3 & 100 & $99 / 1$ & 3 & MMS & 10 & 5 & 94 & 144 & 1.02 & 2.8 \\
\hline D4 & 100 & $99 / 1$ & 3 & MMS & 22 & 5 & 32 & 94 & 1.03 & 2.1 \\
\hline D5 & 100 & $99 / 1$ & 3 & MPDMS & 10 & 4 & 87 & 179 & 1.04 & 1.5 \\
\hline D6 & 25 & $99 / 1$ & 3 & MPDMS & 10 & 3 & 90 & 57 & 1.22 & 10 \\
\hline D7 & 25 & $99 / 1$ & 1.5 & MPDMS & 10 & 3 & 91 & 103 & 1.02 & 2.3 \\
\hline D8 & 25 & $99 / 1$ & 0.75 & MPDMS & 10 & 3 & 79 & 121 & 1.02 & 1.1 \\
\hline D9 & 25 & $99 / 1$ & 0.75 & MPDMS & 10 & $\begin{array}{l}0.1 \\
0.5 \\
3.0\end{array}$ & $\begin{array}{c}8 \\
25 \\
82\end{array}$ & $\begin{array}{c}64 \\
90 \\
127\end{array}$ & $\begin{array}{l}1.04 \\
1.05 \\
1.05\end{array}$ & $\begin{array}{l}0.8 \\
1.0 \\
1.0\end{array}$ \\
\hline D10 & 25 & $99 / 1$ & 0.75 & MPDMS & 10 & $\begin{array}{l}0.5 \\
3.0\end{array}$ & $\begin{array}{l}20 \\
77\end{array}$ & $\begin{array}{c}82 \\
115\end{array}$ & $\begin{array}{l}1.05 \\
1.08\end{array}$ & $\begin{array}{l}1.0 \\
1.2\end{array}$ \\
\hline $\mathrm{D} 11$ & 25 & $99 / 1$ & 0.75 & MPDMS & 10 & 0.6 & 34 & 91 & 1.01 & 0.9 \\
\hline \multicolumn{11}{|c|}{ MMA emulsion polymerization } \\
\hline D12 & 100 & $99 / 1$ & 3 & MPDMS & 10 & 10 & 15 & 101 & 1.02 & 1.9 \\
\hline D13 & 100 & $99 / 1$ & 3 & MPDMS & 20 & 10 & 20 & 108 & 1.02 & 1.5 \\
\hline D14 & 100 & $99 / 1$ & 3 & MPDMS & 30 & 10 & 13 & 73 & 1.02 & 1.3 \\
\hline D15 & 100 & $99 / 1$ & 3 & MPDMS & 30 & 10 & 20 & 84 & 1.06 & 1.1 \\
\hline
\end{tabular}

${ }^{\mathrm{a}}$ All experiments were carried out with $[\mathrm{NaPS}]=0.5 \mathrm{~g} \mathrm{~L}^{-1}$; Silane: 0.1 function $\mathrm{nm}^{-2} ; \mathrm{T}=70{ }^{\circ} \mathrm{C} .{ }^{\mathrm{b}} \mathrm{M}$ stands for monomer. ${ }^{\mathrm{c}} \mathrm{Global}$ concentration of surfactant (i.e. NP30 + SDS). ${ }^{d}$ For PS, the size of the nodules was determined by TEM, except for run D10 where cryo-TEM images were used. For PMMA, the size of the nodules was determined by cryo-TEM exclusively. Note that the swelling of the PS particles by styrene (run D10) or the swelling of the PMMA particles by MMA (runs D12-D15) likely led to overestimated $D_{\mathrm{n}}$ values. ${ }^{\mathrm{e}}$ Calculated from the average diameter of the PS nodules obtained by TEM and using equation 1 , except for run D10 and runs D12 to D15, where $N_{\text {Latex }} / N_{\text {silica }}$ was determined from the manual counting of the number of latex particles and the number of silica particles in the cryo-TEM images, and making the ratio.

Analytical techniques. The latexes (diluted solution deposited on a carbon/formvar-coated copper grid and allowed to evaporate) were observed by transmission electron microscopy (TEM) with a Philips CM120 microscope operating at an accelerating voltage of $80 \mathrm{kV}$ (Centre Technologique des Microstructures (CT $\mu$ ), plate-forme de I'Université Claude Bernard Lyon 1, Villeurbanne, France). In order to provide a more reliable analysis of particle size and morphology, some selected samples of silica/PS dimers and all silica/PMMA samples were observed in their natural hydrated environment 
using cryogenic TEM (cryo-TEM). Thin liquid films of the suspensions were deposited onto on 300 Mesh holey carbon films (AgarScientific, UK) and quench-frozen in liquid ethane using a cryo-plunge workstation (made at LPS Orsay). The specimens were then mounted on a precooled Gatan 626 specimen holder, transferred in the microscope and observed as described previously (at an accelerating voltage of $120 \mathrm{kV})$. The number- and mass-average particle diameter $\left(D_{\mathrm{n}}\right.$ and $D_{\mathrm{w}}$, respectively) as well as the particle-diameter dispersity $\left(D_{\mathrm{w}} / D_{\mathrm{n}}\right)$ were determined using AnalySIS software (Soft Imaging System).

The number ratio of polymer (PMMA or PS) latex particles to silica particles, $N_{\text {Latex }} / N_{\text {silica, }}$ was determined through statistical analysis of the TEM images. Silica and polymer particles show significantly different contrast and can be thus unambiguously identified on the micrographs. However, the procedure for PS was different from that used for PMMA. For the silica/PS dimers, the average diameter of the PS nodules, $D_{\mathrm{n}, \mathrm{PS}}$, was determined by TEM and the ratio $N_{\text {Latex }} / N_{\text {silica }}$ calculated using equation 1:

$$
N_{\text {Latex }} / N_{\text {Silica }}=\left(\frac{C_{\text {PS }}}{C_{\text {Silica }}}\right) \times\left(\frac{\mathrm{d}_{\text {silica }}}{d_{\text {PS }}}\right) \times\left(\frac{D_{\mathrm{n}, \text { silica }}}{D_{\mathrm{n}, \text { PS }}}\right)^{3}
$$

with $C_{\text {Silica }}$ and $C_{P S}\left(g^{-1}\right)$ the silica and PS concentrations, respectively, $d_{\text {silica }}$ and $d_{P S}\left(g^{-3}\right)$ the silica and PS densities (2.0 and 1.05, respectively), and $D_{n, \text { silica }}$ and $D_{\mathrm{n}, \mathrm{PS}}(\mathrm{nm})$ are the diameters of the silica and PS particles determined by TEM, respectively. For the silica/PMMA dimers, due to the likely swelling of PMMA particles by residual MMA, the $N_{\text {Latex }} / N_{\text {silica }}$ ratio was directly determined from the cryo-TEM images by manually counting the number of latex particles and the number of silica particles in the cryo-TEM images, and making the ratio. The morphological yield was calculated with respect to the total number of morphologies present in the sample (i.e. organic/inorganic particles, free polymer particles and free silica seeds).

Charge detection mass spectrometry (CD-MS) experiments were performed on a custom-built charge detection-mass spectrometer coupled with an electrospray source (ESI). This instrument was described in details in previous works. ${ }^{25}$ CD-MS simultaneously and independently measures $m / z$ and $z$ ( $m$ is the molar mass and $z$ the number of charges) for individual ions. Highly charged ions, produced by ESI, pass one at a time through a small metal flight tube attached to a charge-sensitive preamplifier that captures their image current. Once frequency-filtered, amplified and shaped, the primary image current signal is characterized by a double-pulse readout signal. The time between the two pulses corresponds to the flight time required for the ion to traverse the length of the pick-up tube and is a function of the $\mathrm{m} / \mathrm{z}$ ratio of the ion. The amplitude of the signal is proportional to $z$. A primary advantage of the CD-MS measurement approach is the rate of highly charged individual ions that are analyzed. In such conditions, several thousands of ions can be analyzed individually in less than one minute. Calibration in 
charge was performed using a test capacitor that allowed a known amount of charge to be pulsed onto the pick-up tube. The test pulses were generated with a shaping-pulse generator so that the time dependent signal response could be determined as well. The charge of a particle was then directly deduced from this calibration and from the average value of the voltage intensity of the two pulses generated by the particle on the detector. Calibration in mass was performed externally using NIST traceable size standards $(70,100,150,200,250,300,350,400,450$ and $500 \mathrm{~nm}$ PS nanospheres supplied by Polysciences Europe $\mathrm{GmbH}$ ). Aqueous mother solutions of dimer samples were diluted in $\mathrm{H}_{2} \mathrm{O} / \mathrm{MeOH}=1: 1$ solvent mixture at concentration around $10^{14}$ objects $\mathrm{L}^{-1}$ before injection to the ESI source. Mass histograms are built from the collection of a statistically relevant number $N$ of single mass measurement for each sample ( $N>5000$ typically). Samples are a mixture of free polymer particles (PS or PMMA), free silica particles, dimers, bipods, and tripods. The experimental mass histograms are thus fitted with multiple LogNormal distribution fit functions, each corresponding to the individual contribution of free polymer particles, free silica particles, dimers, bipods, and tripods to the overall mass distribution. First, the mass distribution of primary free silica seed particles were measured by CDMS, from which we extracted parameters $x c$ (silica) and $w$ (silica) of LogNormal function (Figure S1). Mean masses of polymer nodules $(x c(p o l))$ were calculated on the basis of the diameter of the objects as determined by TEM and assuming perfectly spherical geometry and densities of 1.05 and 1.19 for PS and PMMA, respectively. These measured and calculated parameters were then combined as a basis for fixing the $x c$ fit parameters for dimers, bipods, and tripods contributions ( $x c(\operatorname{dim}), x c($ bipods), and $x c($ tripods $)$ ). Finally the weight of each contribution was modulated to best fit the experimental distribution (Figures 6, S2 and S3).

\section{Results and discussion}

The original recipe used for several years for the synthesis of various silica/PS clusters was based on a nonionic surfactant (i.e. (poly(ethylene glycol) nonylphenyl ether, with 30 PEG units - referred to as Synperonic ${ }^{\circledR}$ NP30) used in relatively high concentration (ca. $3 \mathrm{~g} \mathrm{~L}^{-1}$ ). However, it is well known that nonionic surfactants usually show poor efficiency in terms of nucleation. As a result, complete conversion was rarely observed. ${ }^{20}$ In addition, even if well-defined clusters could be obtained at low conversion (typically lower than $30 \%$ for $2 \mathrm{~h}$ of reaction), the system was contaminated by a secondary nucleation of pure PS particles finding its origin in NP30 molecules originally present in the monomer droplets and progressively released in the medium as the polymerization proceeds. ${ }^{26}$ On the other hand, using a pure anionic surfactant such as sodium dodecylsulfate (SDS) led, as expected, to higher monomer conversion but the particle morphology appeared less easy to control. ${ }^{27}$ The presence of the non-ionic surfactant indeed seemed to be mandatory and this is likely related to the well-known affinity of 
poly(ethylene oxide)-based macromolecules for silica surfaces. ${ }^{28,29}$ This adsorption probably favors both the localization of monomer molecules owing to the phenyl group of NP30 and the growth of the polymer chains at the surface.

For the synthesis of pure polymer particles, one way to improve the nucleation in non-ionic surfactantbased systems consists in the addition of a few percent of an anionic surfactant such as SDS to the nonionic one. ${ }^{30-33}$ This strategy is widely used in the industry to take advantage of the complementary roles of both types of surfactants. The anionic surfactant will provide efficient nucleation, while the non-ionic one will ensure the stabilization of the latex over a broad range of conditions (stability against freezethaw, shear or in the presence of polyelectrolytes).

We recently showed that the polymerization recipe could be optimized for the synthesis of multipods/silica clusters by the substitution of $5 \mathrm{wt} \%$ of NP30 for SDS. ${ }^{22}$ In this case, large amounts of either tetrapods or hexapods (i.e. four, respectively six, PS nodules surrounding one silica core) were prepared with yields over $80 \%$. In the present paper, the use of the mixed surfactant system was extended to the synthesis of silica/polymer (either PS or PMMA) dimers with the aim of producing large amounts of materials (at the gram scale). In the original studies, ${ }^{21}$ silica/PS dimers were obtained using NP30 as a surfactant and $80 \mathrm{~nm}$ silica seeds $\left(10 \mathrm{~g} \mathrm{~L}^{-1}\right)$ obtained by the Stöber process and surfacemodified with MMS with a nominal surface density of 0.1 function $\mathrm{nm}^{-2}$. MMS grafting was performed in hydroalcoholic media. In the present work, we put a special focus on simplifying the process to allow broad use of this strategy and an easy access to gram-scale products. A commercial aqueous silica dispersion was thus used instead of home-made silica seeds, and MMS grafting was performed directly in water in the presence of NP30 and/or SDS instead of using a water/ethanol mixture requiring further separation/redispersion steps. Therefore, the second role of the surfactant(s) was to help the dispersion of MMS molecules in water. ${ }^{27}$

\section{Synthesis of silica/PS heterodimers}

A first series of experiments was carried out with various surfactant systems using the $80 \mathrm{~nm}$ silica seeds $\left(10 \mathrm{~g} \mathrm{~L}^{-1}\right)$ surface-modified with MMS $\left(0.1\right.$ function $\left.\mathrm{nm}^{-2}\right)$. Sodium persulfate was used as initiator and various mixtures of NP30/SDS as surfactants (runs D1-D3, Table 1 and Figure 1). 


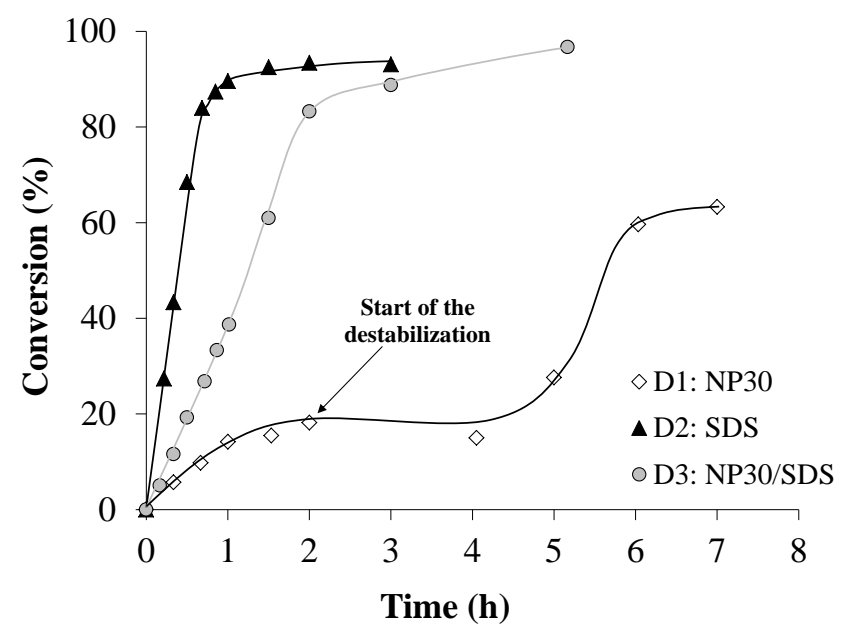

Figure 1: Evolution of monomer conversion versus time for different surfactant systems in the synthesis of silica/PS heterodimers: $(\diamond)$ NP30 (run D1), ( $\mathbf{\Delta}$ ) SDS (run D2) and (0) NP30/SDS (99/1 wt/wt, run D3). See Table 1 for detailed experimental conditions.

As expected, the rate of styrene consumption was greatly increased when SDS was used together with NP30 (run D3), the polymerization being complete in $5 \mathrm{~h}$, while for the same polymerization time only ca. $25 \%$ of styrene was converted in the absence of SDS (run D1). In the latter case, secondary nucleation occurred (evidenced by the increase of the polymerization rate at ca. $4.5 \mathrm{~h}$ corresponding to the formation of a new crop of particles, and by TEM observations) and the latex was not stable after 2 hours of polymerization. As expected, using pure SDS (run D2) led to even faster polymerization but to the detriment of the morphology control as in this case the nucleation was too efficient (even if the initial SDS concentration was lowered to $2 \mathrm{~g} \mathrm{~L}^{-1}$ ). An accurate statistical analysis of particle morphologies using TEM images remained quite difficult, the particles being prone to aggregate on the TEM grids. This was actually the case for most of the TEM observations performed throughout this work. The value of the ratio $N_{\text {Latex }} / N_{\text {silica }}$ was consequently chosen to discuss the outcomes of an experiment. In the case of run D2 (pure SDS), a few dimers were observed together with a large amount of free PS particles leading to $N_{\text {Latex }} / N_{\text {silica }}=3.6$ (Figure 2b). The $N_{\text {Latex }} / N_{\text {silica }}$ ratio decreased to 2.8 for NP30/SDS 99/1 (wt/wt) with mainly tetrapods and tripods present (Figure 2c). The influence of the NP30/SDS weight ratio $(99 / 1 ; 98 / 2 ; 95 / 5 ; 90 / 10)$ was also studied (data not shown). A 99/1 ratio appeared as the best compromise between a high polymerization rate and a reduced number of free polymer particles (the proportion of which increased with the SDS fraction). 


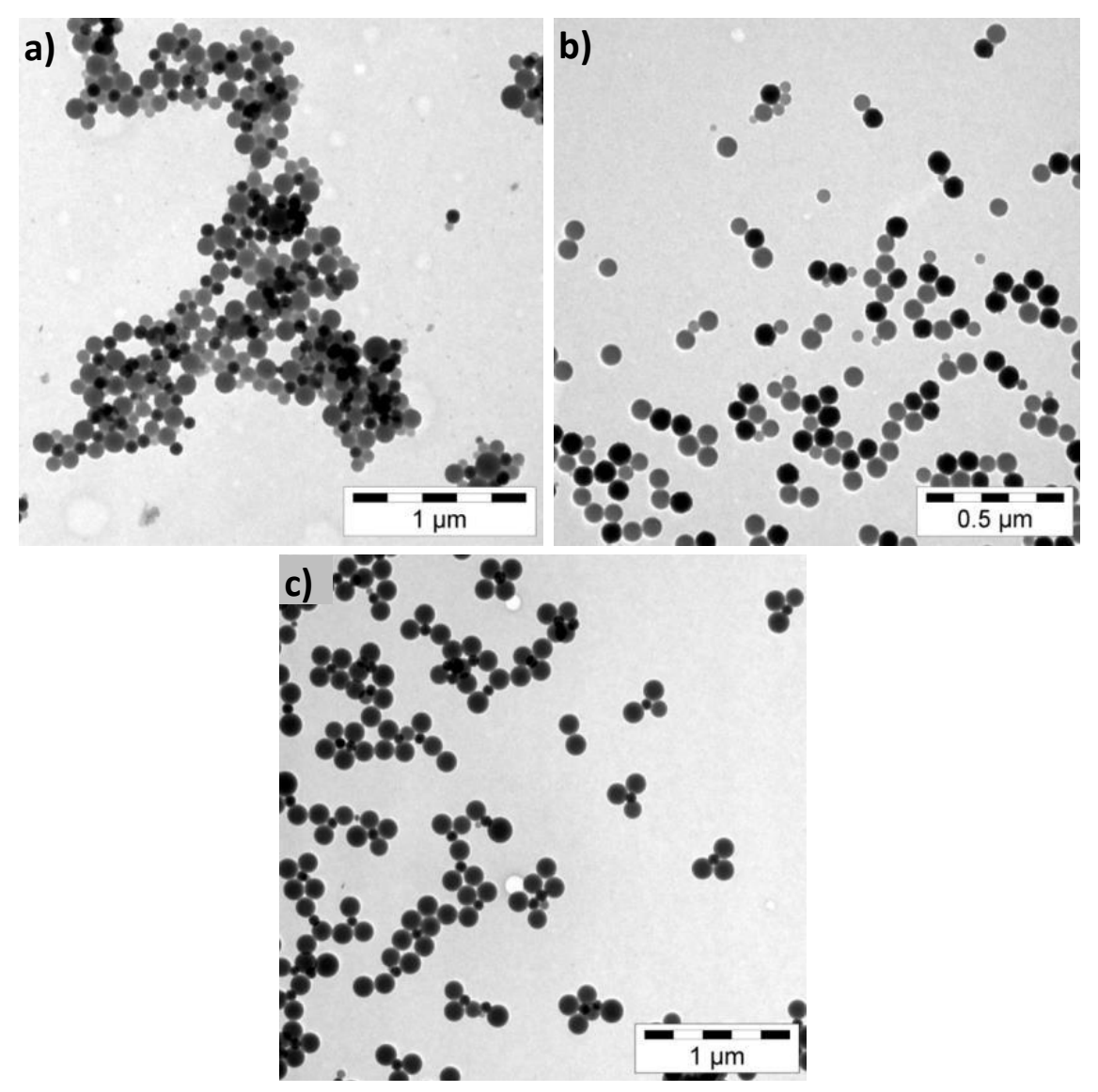

Figure 2: TEM images of the silica/PS latexes obtained for different surfactant systems in the synthesis of silica/PS heterodimers: a) NP30 (run D1, time $=1 \mathrm{~h}$, before destabilization), b) SDS (run D2, time $=3 \mathrm{~h}$ ) and c) NP30/SDS (99/1 wt/wt, run D3, time $=2 \mathrm{~h}$ ). See Table 1 for detailed experimental conditions.

To decrease the $N_{\text {Latex }} / N_{\text {silica }}$ ratio, one experiment was then carried out with a higher silica concentration (run D4, [Silica] $=22 \mathrm{~g} \mathrm{~L}^{-1}$, Table 1). Indeed, increasing the number of silica seeds while keeping the same amount of styrene was expected to lead to the formation of less PS nodules per silica particle. $N_{\text {Latex }} / N_{\text {silica }}$ was effectively decreased to 2.1 with the formation of some dissymmetrical particles, together with a majority of dipods and a few tripods. In addition, the conversion was limited to $\mathrm{ca} .30 \%$ conversion, the latex becoming quickly unstable, preventing any reliable statistical analysis.

As an increase in silica concentration led to a loss of colloidal stability, another strategy was then envisioned to reduce the number of PS nodules per silica seed (i.e. the amount of tri- and/or tetrapods), consisting in the use of a monofunctional silane, MPDMS, instead of MMS (run D5, see Table 1 and Figure 3). Indeed, for the same initial nominal amount (i.e. 0.1 function $\mathrm{nm}^{-2}$ ), the grafting density was expected to be easier to control with MPDMS as a trifunctional silane such as MMS may react with the silica surface (as expected) but also with other silane molecules. ${ }^{34}$ The use of MPDMS effectively led to the formation of fewer particles and the kinetics was consequently slower (data not shown). At the end of the polymerization (i.e. after $4 \mathrm{~h}$ corresponding to $87 \%$ conversion), the ratio $N_{\text {Latex }} / N_{\text {silica }}$ was 
effectively reduced to 1.5 and snowman-like dissymmetrical particles with a PS lobe close to $180 \mathrm{~nm}$ were observed on TEM pictures with a yield of ca. 60\% (red arrows, Figure 3d). However, other morphologies such as dipods or very low amounts of free silica or PS particles were also observed. Note that in both cases dimpled particles corresponding to detached PS nodules could be seen at low monomer-to-polymer conversions (see black arrows in Figure 3a or 3c). These peculiar morphologies are discussed later.

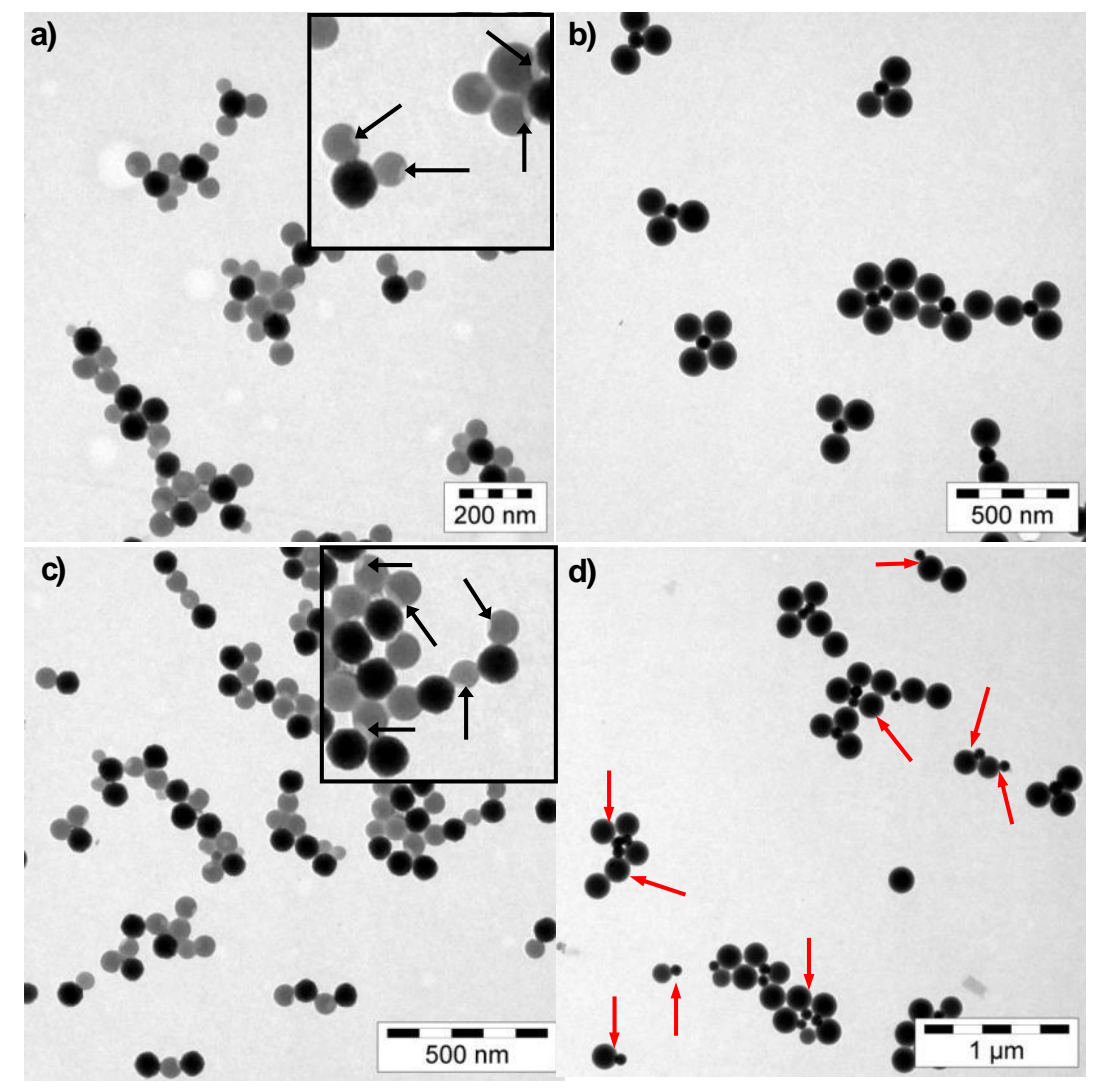

Figure 3: TEM images of the silica/PS latexes obtained using different silanes. MMS (run D3): (a) 20 min, $12 \%$ conversion, (b) 2h, 83\% conversion; and MPDMS (run D5): (c) $20 \mathrm{~min}, 6 \%$ conversion, (d) 4h, 87\% conversion. See Table 1 for detailed experimental conditions. In images a) and c), arrows point to the PS dimpled particles. In image d), red arrows point to the silica/PS snowman-like particles.

Besides the production of these snowman-like silica/PS particles, the production of dumbbells, i.e. with a PS lobe of the same size than the silica one $(80 \mathrm{~nm})$, was also envisioned. We thus played with the concentration of both styrene and the surfactants to reduce the size of the PS nodules. First, styrene concentration was divided by 4 (i.e. $25 \mathrm{~g} \mathrm{~L}^{-1}$ ) keeping the same concentration of surfactants $\left(3 \mathrm{~g} \mathrm{~L}^{-1}\right.$ ) (run D6, Table 1). The size of the PS nodule was effectively decreased $\left(D_{n, P S}=57 \mathrm{~nm}\right)$ but the surfactant concentration was too high for this amount of styrene and many free PS particles were formed $\left(N_{\text {Latex }} / N_{\text {silica }}=10\right)$ together with multipods-like structures with very small PS lobes $\left(D_{\mathrm{n}, \mathrm{PS}}=34 \mathrm{~nm}\right)$. 
Consequently, the surfactant concentration was reduced to $1.5 \mathrm{~g} \mathrm{~L}^{-1}$ (run D7, Table 1 ) and finally to 0.75 $\mathrm{g} \mathrm{L}^{-1}$ (run D8, Table 1) which effectively induced in both cases a lower number of particles nucleated $\left(N_{\text {Latex }} / N_{\text {Silica }}=2.3\right.$ and 1.1, respectively $)$ and consequently a higher diameter for the PS lobe $\left(D_{\mathrm{n}, \mathrm{PS}}=103\right.$ $\mathrm{nm}$ and $121 \mathrm{~nm}$, respectively, Table 1 and Figure 4). Again, an accurate statistical analysis of the TEM pictures remained quite difficult, the particles being prone to aggregation on the TEM grids. The size of the PS lobe in run D8 $\left(N_{\text {Latex }} / N_{\text {silica }}=1.1\right)$ was higher than targeted but it is worth mentioning that the monomer conversion remained quite high (79\%).

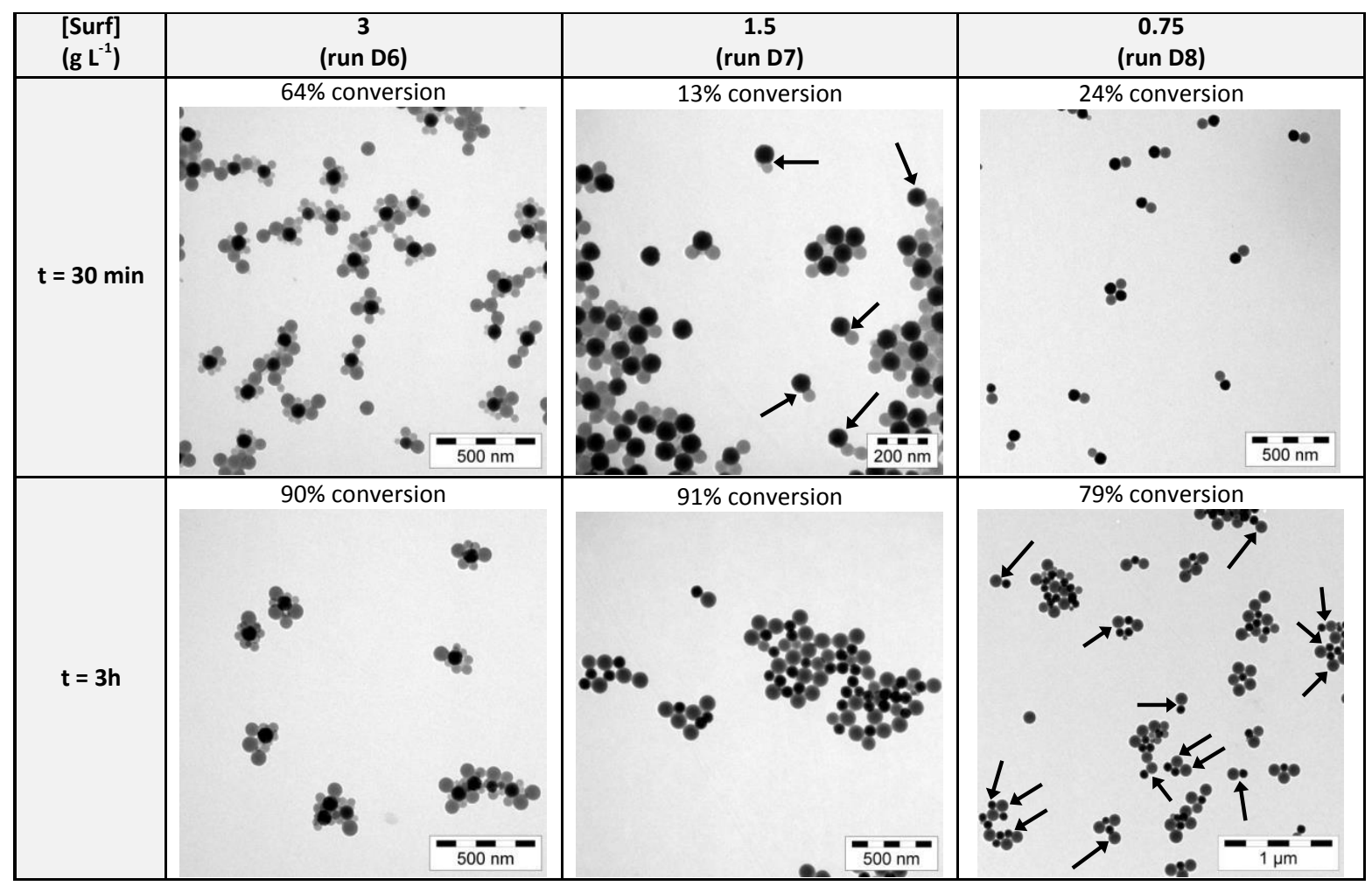

Figure 4: TEM images of the silica/PS latexes obtained using different concentrations of the NP30/SDS mixture (99/1 wt/wt). [Surf] = $3 \mathrm{~g} \mathrm{~L}^{-1}$ (run D6); [Surf] = $1.5 \mathrm{~g} \mathrm{~L}^{-1}$ (run D7); [Surf] = $0.75 \mathrm{~g} \mathrm{~L}^{-1}$ (run D8). See Table 1 for detailed experimental conditions. Arrows point to the silica/PS dissymmetrical particles.

In all the recipes presented so far the obtained dimers showed a snowman-like morphology at high conversion, the PS lobe being systematically bigger than the silica seed. To obtain dumbbell-like dimers one strategy consists in quenching the polymerization when the PS lobe is close to $80 \mathrm{~nm}$. To determine the moment when this size was reached, the evolution of particle morphology was monitored as a function of conversion for a D8-type recipe (run D9, Figure 5). To provide a more reliable analysis of particle morphology, the hybrid particles were observed by cryo-TEM in their hydrated environment. As expected, the size of the PS lobe increased with conversion, and dumbbell-like particles were obtained after 30 min corresponding to $25 \%$ conversion $\left(D_{\mathrm{n}, \mathrm{Ps}}=90 \mathrm{~nm}, D_{\mathrm{w}} / D_{\mathrm{n}}=1.05\right)$. In this case, a statistical 
analysis based on 100 particles gave $53 \%$ dimers, $8 \%$ dipods, less than $1 \%$ tetrapods, and $38 \%$ isolated silica spheres. This recipe actually offers the possibility to vary the morphology from mainly snowmanlike (at low and high conversions, silica being initially the largest lobe, and finally the smallest one) to dumbbell-like dimers at an intermediate conversion.
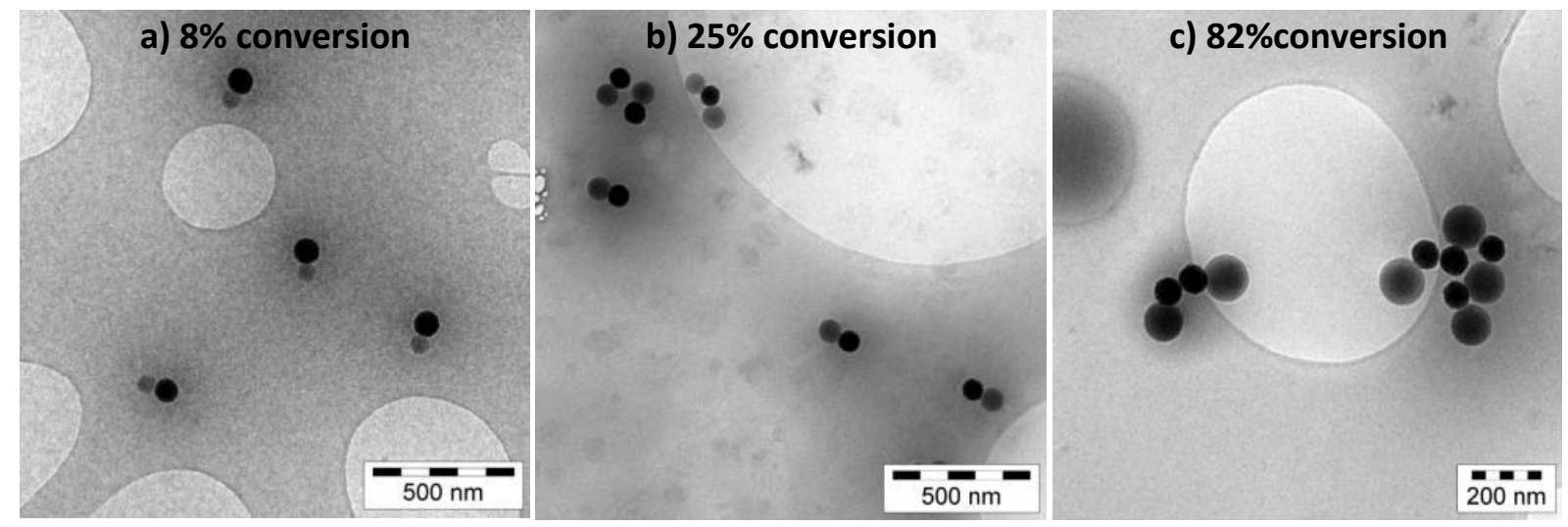

Figure 5: Cryo-TEM images showing the evolution of particle morphology with conversion for the synthesis of silica/PS dimers using a D8-type recipe (run D9, see Table 1 for detailed experimental conditions): a) 8\% conversion: $D_{\mathrm{n}, \mathrm{Ps}}=64 \mathrm{~nm}, D_{\mathrm{w}} / D_{\mathrm{n}}=1.04$, b) $25 \%$ conversion, $D_{\mathrm{n}, \mathrm{Ps}}=90 \mathrm{~nm}, D_{\mathrm{w}} / D_{\mathrm{n}}=1.05$, c) $82 \%$ conversion, $D_{\mathrm{n}, \mathrm{Ps}}=$ $127 \mathrm{~nm}, D_{\mathrm{w}} / D_{\mathrm{n}}=1.05$.

With the goal of obtaining the clearest picture of the dispersed state of the system and thus clearing any doubt about the sample composition in terms of cluster morphology, further analyses were undertaken using the emerging technique of charge detection mass spectrometry (CD-MS). ${ }^{23,24}$ The CDMS-based analyses, thanks to the single-ion measuring approach, provide a direct measurement of molar mass distributions along with the charge distributions of nanoparticles. For heterogeneous samples (as in the current case), this technique appears to be efficient to fathom the relative composition of the mixture. ${ }^{24,35} \mathrm{CD}-\mathrm{MS}$ measurements analyze up to several thousand particles and could thus provide a picture of the whole sample complementary to the information brought by TEM. A comparison of both techniques was performed on the sample obtained at medium (20\%) and high (77\%) conversion for a D8-type recipe (run D10, Table 2). The ion population corresponding to the resulting colloidal clusters was deconvoluted in a sum of several sub-population contributions as described in Experimental Section and illustrated in Figure 6 for the low conversion sample. Both techniques provide a similar analysis of the sample composition at a given styrene conversion time. For instance, at $20 \%$ styrene conversion, proportion of dimers of $52.3 \%$ and $55 \%$ are found in TEM and CD-MS, respectively, thus demonstrating the majority proportion reached for the targeted cluster type. In addition, both analyses exhibited the same trend while extending the styrene conversion time. The proportion of free silica significantly decreased (from $20 \%$ to $8.5 \%$ in TEM and from $12 \%$ to $2 \%$ in CDMS) and the proportion of dipods and tripods increased slightly relatively to that of dimers. 
Table 2: Proportion of the different morphologies encountered in the silica/PS heterodimers batch (run D10) and in the silica/PMMA heterodimers batch (run D15) as determined by TEM and CD-MS ${ }^{a}$

\begin{tabular}{|c|c|c|c|c|c|c|c|c|c|c|}
\hline \multicolumn{11}{|c|}{ Silica/PS heterodimers } \\
\hline $\begin{array}{l}\text { Reaction } \\
\text { time (min) }\end{array}$ & \multicolumn{5}{|c|}{30} & \multicolumn{5}{|c|}{180} \\
\hline $\begin{array}{l}\text { Styrene } \\
\text { conv. (\%) }\end{array}$ & \multicolumn{5}{|c|}{20} & \multicolumn{5}{|c|}{77} \\
\hline$D_{\mathrm{n}, \mathrm{Ps}}(\mathrm{nm})$ & \multirow{2}{*}{\multicolumn{5}{|c|}{$\begin{array}{c}82 \\
1.05\end{array}$}} & \multirow{2}{*}{\multicolumn{5}{|c|}{$\begin{array}{l}115 \\
1.08\end{array}$}} \\
\hline$D_{\mathrm{w}} / D_{\mathrm{n}}$ & & & & & & & & & & \\
\hline $\begin{array}{c}\% \\
\text { Morphology }^{b}\end{array}$ & Dimers & Dipods & Tripods & $\begin{array}{c}\text { Free } \\
\text { PS }\end{array}$ & $\begin{array}{l}\text { Free } \\
\text { silica }\end{array}$ & Dimers & Dipods & Tripods & $\begin{array}{l}\text { Free } \\
\text { PS }\end{array}$ & $\begin{array}{l}\text { Free } \\
\text { silica }\end{array}$ \\
\hline TEM & 52.3 & 18 & 7.7 & 2 & 20 & 48.5 & 23.5 & 14 & 5.5 & 8.5 \\
\hline CD-MS & 55 & 15 & 13 & 5 & 12 & 46 & 26 & 24 & 2 & 2 \\
\hline \multicolumn{11}{|c|}{ Silica/PMMA heterodimers } \\
\hline $\begin{array}{l}\text { Reaction } \\
\text { time (min) }\end{array}$ & \multicolumn{5}{|c|}{10} & \multicolumn{5}{|c|}{20} \\
\hline $\begin{array}{c}\text { MMA conv. } \\
(\%)\end{array}$ & \multicolumn{5}{|c|}{20} & \multicolumn{5}{|c|}{48} \\
\hline$D_{n, \text { PMMA }}(\mathrm{nm})$ & \multicolumn{5}{|c|}{84} & \multicolumn{5}{|c|}{124} \\
\hline$D_{\mathrm{w}} / D_{\mathrm{n}}$ & \multicolumn{5}{|c|}{1.06} & \multicolumn{5}{|c|}{1.12} \\
\hline $\begin{array}{c}\% \\
\text { Morphology }\end{array}$ & Dimers & Dipods & Tripods & \begin{tabular}{|c|} 
Free \\
PMMA
\end{tabular} & $\begin{array}{l}\text { Free } \\
\text { silica }\end{array}$ & Dimers & Dipods & Tripods & \begin{tabular}{|c|} 
Free \\
PMMA
\end{tabular} & $\begin{array}{l}\text { Free } \\
\text { silica }\end{array}$ \\
\hline TEM & 55 & 28 & 5 & 2 & 10 & 57 & 27 & 3 & 1 & 12 \\
\hline CD-MS & 49 & 19 & 19 & 5 & 8 & 47 & 22 & 17 & 13 & $<1$ \\
\hline
\end{tabular}

${ }^{\mathrm{a}}$ See Table 1 for details on runs D10 and D15; ${ }^{\mathrm{b}} \%$ with respect to all morphologies.

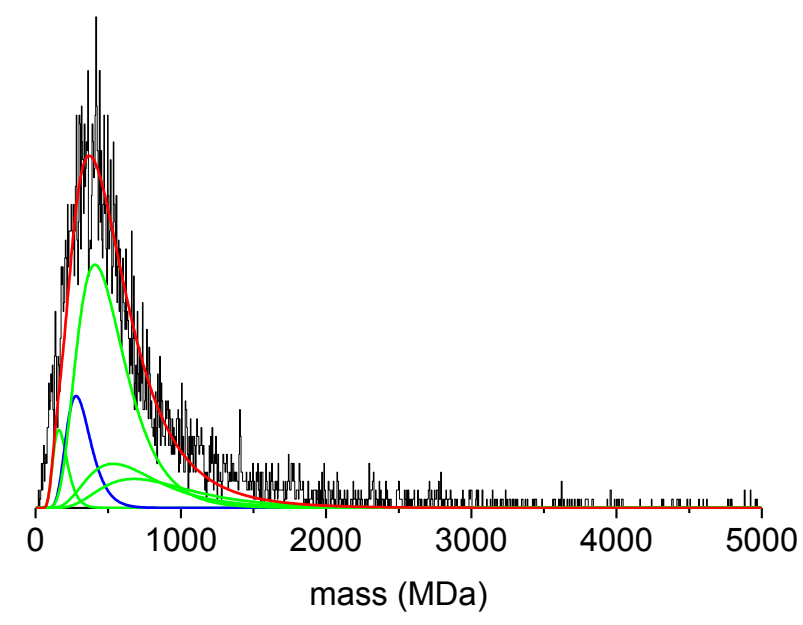

Figure 6. Mass distribution of silica/PS dimers sample measured by CDMS (black edged circles) at $20 \%$ monomer conversion ( $t=30 \mathrm{~min}$ ). The blue line corresponds to the possible contribution of free silica particles to the overall distribution, green lines are contributions of free PS particle, dimers, dipods, and tripods, respectively, while the red line is the sum of all the contributions. 


\section{Synthesis of silica/PMMA heterodimers}

To show the versatility of our synthetic strategy, the formation of silica/PMMA dimers was also undertaken. Such heterodimers were recently obtained in our group using a new and different strategy which relies on the adsorption of a bicationic initiator on the silica surface, ${ }^{36}$ and both snowman- and dumbbell-like silica/polymer clusters were obtained through emulsion polymerization of MMA or styrene. The first experiment was carried out using experimental conditions similar to those adopted for styrene, targeting low conversion to reach dumbbell-like morphologies (run D12, Table 1). Due to the higher water solubility and reactivity of MMA leading to a more efficient nucleation, the number of free PMMA particles was higher than expected $\left(N_{\text {Latex }} / N_{\text {silica }}=1.9\right)$. The concentration of silica was thus increased to $20 \mathrm{~g} \mathrm{~L}^{-1}$ (run D13) and then to $30 \mathrm{~g} \mathrm{~L}^{-1}$ (run D14). In both cases the $N_{\text {Latex }} / N_{\text {silica }}$ ratio was effectively lowered, reaching 1.3 in the latter case (Table 1). This ratio could not be decreased further, higher silica concentration leading to colloidal stability issues. Many dimers could be clearly observed by cryo-TEM in run D14 (Figure 7), statistical analyses indicating $47 \%$ of dimers, $35 \%$ of dipods, $2 \%$ of tripods, $2 \%$ of free PMMA particles and $14 \%$ of free silica particles. Both statistical analyses of TEM images and CD-MS measurements were performed on a D14-type recipe (run D15). The data shown in Table 2 again indicate a relatively good agreement between the two techniques, with however significant differences in the case of tripods (Figure S3).

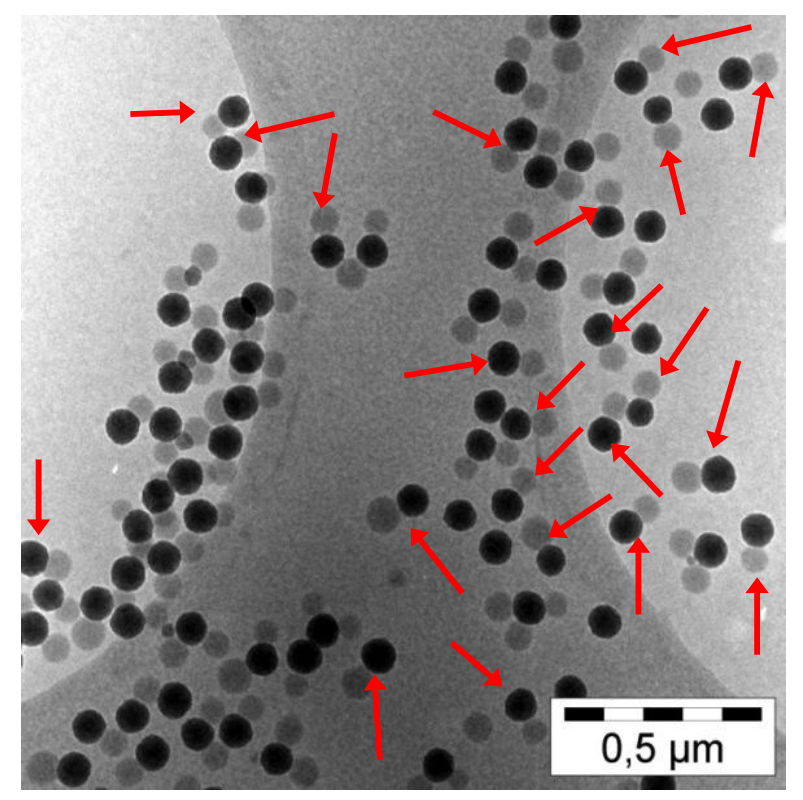

Figure 7: Cryo-TEM image of the silica/PMMA latex obtained using $30 \mathrm{~g} \mathrm{~L}^{-1}$ of silica (run D14, 13\% conversion). See Table 1 for detailed experimental conditions. Arrows point at some of the dimers. 


\section{Formation of dimpled polymer particles}

One very interesting phenomenon was observed with the samples containing residual styrene. As evidenced by the TEM images of run D8 at low conversion (Figure 3a and 3c), the link between the silica and PS lobes seems to be quite weak as free dimpled PS particles could be observed. In order to manipulate these objects, we tried to remove styrene by gentle stirring under nitrogen bubbling of the dispersion containing dumbbell-like morphologies (run D11, 25\% conversion). The latex became unstable and a phase separation occurred after 4 days. Whereas the bottom layer was essentially constituted of silica particles, TEM observation of the top layer (Figure 8) showed the presence of free dimpled PS particles, which took off from the silica surface. This separation of the PS nodule from the silica seed is very likely related to weak adhesion forces, essentially secured by a few PS chains covalently bonded to the silica surface and directly dependent on the low grafting density of the copolymerizable silane. Besides, these polymer chains should be quite mobile in the context of a polymer nodule swollen with unreacted monomer ( $25 \%$ conversion), promoting their disentanglement from the PS nodule ones. This makes the assembly silica seed/PS nodule quite fragile with regard to shear stress as potentially occurring during mechanical stirring or centrifugation stage. On the other hand, the presence of a notch on the PS particles is objective evidence that particle growth occurred at the silica surface. Occasionally, PS particles with two dimples, originating from dipods silica/PS clusters could be observed. Dimpled particles are a very interesting class of colloidal objects which can be used as building block in various architectures such as new colloidal molecules or low-symmetry photonic crystals. ${ }^{37-41}$ In comparison with the strategies depicted in the literature, our synthetic approach is very simple and already efficient at the gram scale; nevertheless it is restricted to the fabrication of polymer particles mainly bearing a single circular notch.

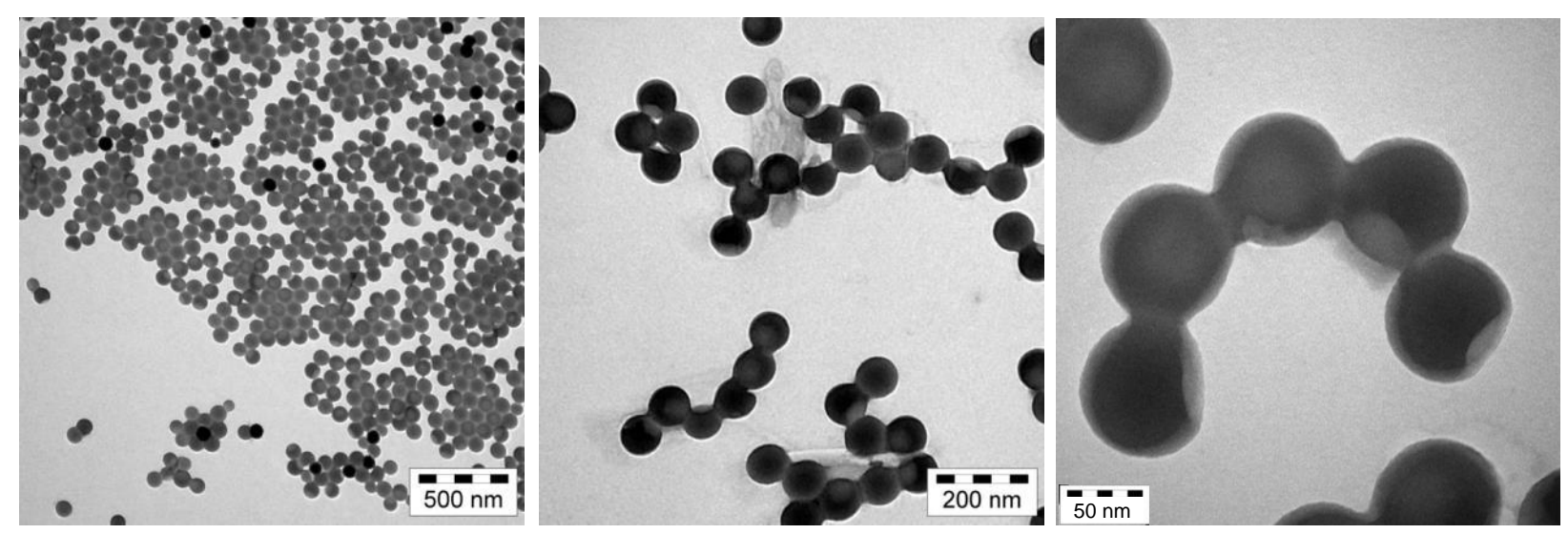

Figure 8: TEM images of the PS particles obtained after one week of gentle stirring (run D11, see Table 1 for detailed experimental conditions). Only the supernatant was analyzed. 


\section{Conclusions}

Silica/PS heterodimers have been successfully obtained by emulsion polymerization using silica seeds pre-functionalized by a reactive silane. Aiming at a scalable and robust process allowing the formation of large amounts of dimers, a commercial silica sol was used, and the silane grafting reaction was successfully performed directly in the mixture of surfactants subsequently used for the polymerization instead of in alcoholic media, thus avoiding time-consuming purification steps. The influence of various experimental parameters (silica content, nature and concentration of both the surfactant and the alkoxysilane) on the formation of silica/PS dimers was investigated. A fine tuning of the silica, styrene and surfactants concentrations using the monofunctional silane MDPMS led to the formation of either dumbbell- or snowman-like dimers, depending on monomer conversion. The typical morphological yield reached $60 \%$ with respect to all the morphologies present in the sample. The synthesis of silica/PMMA dumbbell-like dimers was also successful. For both types of heterodimers, CD-MS revealed to be a powerful technique for the characterization of the silica/polymer clusters, complementary to and in good agreement with TEM analyses. This emerging technique provided the true mass distribution and consequently the cluster composition in terms of morphologies, thus clearing any doubt about the clusters' structure. Finally, this system also proved to be an efficient way to access to dimpled polymer particles.

\section{Acknowledgements}

The support of the Agence Nationale pour la Recherche is gratefully acknowledged (ToCoMo project contract \# ANR-07-BLAN-0271; PONAME project - contract \# ANR-11-PDOC-032-01).

\section{Electronic Supplementary Information}

Chemical structures of the main chemicals used in the study; Mass distribution of free silica particles measured by CD-MS and the corresponding LogNormal fitted distribution; Mass distribution of silica/PS dimers sample measured by CD-MS at $77 \%$ monomer conversion; Mass distribution of silica/PMMA dimers sample measured by CD-MS at two monomer conversions. 


\section{References}

1. Duguet, E.; Désert, A.; Perro, A.; Ravaine, S. Chem. Soc. Rev. 2011, 40, 941-960.

2. Li, F.; Josephson, D. P.; Stein, A. Angew. Chem. Int. Ed. 2011, 50, 360-388.

3. Jones, M. R.; Mirkin, C. A. Nature 2012, 491, 42-43.

4. Wang, Y.; Wang, Y.; Breed, D. R.; Manoharan, V. N.; Feng, L.; Hollingsworth, A. D.; Weck, M.; Pine, D. J. Nature 2012, 491, 51-55.

5. Sacanna, S.; Pine, D. J. Curr. Opin. Colloid Interface Sci. 2011, 16, 96-105.

6. Pawar, A., B.; Kretzschmar, I. Macromol. Rapid Commun. 2010.

7. Cho, Y.-S.; Yi, G.-R.; Lim, J.-M.; Kim, S.-H.; Manoharan, V. N.; Pine, D. J.; Yang, S.-M. J. Am. Chem. Soc. 2005, 127, 15968-15975.

8. Qiang, W.; Wang, Y.; He, P.; Xu, H.; Gu, H.; Shi, D. Langmuir 2008, 24, 606-608.

9. Nagao, D.; Hashimoto, M.; Hayasaka, K.; Konno, M. Macromol. Rapid Commun. 2008, 29, 14841488.

10. Nagao, D.; van Kats, C. M.; Hayasaka, K.; Sugimoto, M.; Konno, M.; Imhof, A.; van Blaaderen, A. Langmuir 2010, 26, 5208-5212.

11. Tang, C.; Zhang, C.; Liu, J.; Qu, X.; Li, J.; Yang, Z. Macromolecules 2010, 43, 5114-5120.

12. Yin, Y.; Zhou, S.; You, B.; Wu, L. J. Polym. Sci., Part A: Polym. Chem. 2011, 49, 3272-3279.

13. Sacanna, S.; Rossi, L.; Pine, D. J. J. Am. Chem. Soc. 2012, 134, 6112-6115.

14. Hu, J.; Zhou, S.; Sun, Y.; Fang, X.; Wu, L. Chem. Soc. Rev. 2012, 41, 4356-4378.

15. Liu, B.; Zhang, W.; Zhang, D.; Yang, X. J. Colloid Interface Sci. 2012, 385, 34-40.

16. Ji, X.; Wang, M.; Ge, X.; Liu, H. Langmuir 2013, 29, 1010-1016.

17. Yabu, H.; Ohshima, H.; Saito, Y. ACS Appl. Mater. Interfaces 2014, 6, 18122-18128.

18. Tian, L.; Zhang, B.; Li, W.; Li, X.; Fan, X.; Jia, X.; Zhang, H.; Zhang, Q. RSC Adv. 2014, 4, 2715227158.

19. Lv, L.-P.; Zhao, Y.; Zhou, H.-X.; Landfester, K.; Crespy, D. Polymer 2014, 55, 715-720.

20. Perro, A.; Duguet, E.; Lambert, O.; Taveau, J. C.; Bourgeat-Lami, E.; Ravaine, S. Angew. Chem. Int. Ed. 2009, 48, 361-365.

21. Reculusa, S.; Poncet-Legrand, C.; Perro, A.; Duguet, E.; Bourgeat-Lami, E.; Mingotaud, C.; Ravaine, S. Chem. Mater. 2005, 17, 3338-3344.

22. Désert, A.; Chaduc, I.; Fouilloux, S.; Taveau, J.-C.; Lambert, O.; Lansalot, M.; Bourgeat-Lami, E.; Thill, A.; Spalla, O.; Ravaine, S.; Duguet, E. Polym. Chem. 2012, 3, 1130-1132.

23. Fuerstenau, S. D.; Benner, W. H. Rapid Commun. Mass Spectrom. 1995, 9, 1528-1538.

24. Doussineau, T.; Désert, A.; Lambert, O.; Taveau, J.-C.; Lansalot, M.; Dugourd, P.; Bourgeat-Lami, E.; Ravaine, S.; Duguet, E.; Antoine, R. J. Phys. Chem. C 2015, 119, 10844-10849.

25. Doussineau, T.; Yu Bao, C.; Clavier, C.; Dagany, X.; Kerleroux, M.; Antoine, R.; Dugourd, P. Rev. Sci. Instrum. 2011, 82, 084104.

26. Özdeğer, E.; Sudol, E. D.; El-Aasser, M. S.; Klein, A. J. Polym. Sci., Part A: Polym. Chem. 1997, 35, 3813-3825.

27. Bourgeat-Lami, E.; Insulaire, M.; Reculusa, S.; Perro, A.; Ravaine, S.; Duguet, E. J. Nanosci. Nanotechnol. 2006, 6, 432-444.

28. Mathur, S.; Moudgil, B. M. J. Colloid Interface Sci. 1997, 196, 92-98.

29. Tiberg, F.; Brinck, J.; Grant, L. Curr. Opin. Colloid Interface Sci. 1999, 4, 411-419.

30. Chu, H.-H.; Piirma, I. Polym. Bull. 1989, 21, 301-308.

31. Chern, C. S.; Lin, S. Y.; Chen, L. J.; Wu, S. C. Polymer 1997, 38, 1977-1984.

32. Colombié, D.; Sudol, E. D.; El-Aasser, M. S. Macromolecules 2000, 33, 7283-7291. 
33. Colombié, D.; Sudol, E. D.; El-Aasser, M. S. Macromolecules 2000, 33, 4347-4353.

34. Negrete Herrera, N.; Letoffe, J.-M.; Reymond, J.-P.; Bourgeat-Lami, E. J. Mater. Chem. 2005, 15, 863-871.

35. Doussineau, T.; Paletto, P.; Dugourd, P.; Antoine, R. J. Am. Soc. Mass Spectrom. 2015, 26, 7-13.

36. Parvole, J.; Chaduc, I.; Ako, K.; Spalla, O.; Thill, A.; Ravaine, S.; Duguet, E.; Lansalot, M.; BourgeatLami, E. Macromolecules 2012, 45, 7009-7018.

37. Champion, J. A.; Katare, Y. K.; Mitragotri, S. Proc. Natl. Acad. Sci. U.S.A. 2007, 104, 11901-11904.

38. Sacanna, S.; Irvine, W. T. M.; Chaikin, P. M.; Pine, D. J. Nature 2010, 464, 575-578.

39. Kim, S.-H.; Hollingsworth, A. D.; Sacanna, S.; Chang, S.-J.; Lee, G.; Pine, D. J.; Yi, G.-R. J. Am. Chem. Soc. 2012, 134, 16115-16118.

40. Désert, A.; Hubert, C.; Fu, Z.; Moulet, L.; Majimel, J.; Barboteau, P.; Thill, A.; Lansalot, M.; Bourgeat-Lami, E.; Duguet, E.; Ravaine, S. Angew. Chem., Int. Ed. 2013, 52, 11068-11072.

41. Wang, Y.; Wang, Y.; Zheng, X.; Yi, G.-R.; Sacanna, S.; Pine, D. J.; Weck, M. J. Am. Chem. Soc. 2014, 136, 6866-6869. 